\title{
Reverse Thrust Aerodynamics of Variable Pitch Fans
}

\author{
Tim S. Williams* \\ Whittle Laboratory, \\ University of Cambridge, \\ $1 \mathrm{JJ}$ Thomson Avenue, \\ Cambridge CB3 ODY, UK \\ Email: tsw30@cam.ac.uk \\ Cesare A. Hall \\ Whittle Laboratory, \\ University of Cambridge, \\ 1 JJ Thomson Avenue, \\ Cambridge CB3 ODY, UK \\ Email: cah1003@cam.ac.uk
}

\begin{abstract}
Variable pitch fans are of interest for future low pressure ratio fan systems since they provide improved operability relative to fixed pitch fans. If they can also be re-pitched such that they generate sufficient reverse thrust they could eliminate the engine drag and weight penalty associated with bypass duct thrust reversers. This paper sets out to understand the details of the $3 D$ fan stage flow field in reverse thrust operation.

The study uses the Advanced Ducted Propulsor variable pitch fan test case, which has a design fan pressure ratio of 1.29. Comparison with spanwise probe measurements show that the computational approach is valid for examining the variation of loss and work in the rotor in forward thrust. The method is then extended to a reverse thrust configuration using an extended domain and appropriate boundary conditions.

Computations, run at two rotor stagger settings, show that the spanwise variation in relative flow angle onto the rotor aligns poorly to the rotor inlet metal angle. This leads to two dominant rotor loss sources: one at the tip associated with positive incidence, and the second caused by negative incidence at lower span fractions. The second loss is reduced by opening the rotor stagger setting, and the first increases with rotor suction surface Mach
\end{abstract}

${ }^{*}$ Corresponding author. 
number. The higher mass flow at more open rotor settings provide higher gross thrust, up to $49 \%$ of the forward take-off value, but is limited by the increased loss at high speed.

\section{Nomenclature}

A Area, $\mathrm{m}^{2}$

c Blade section aerodynamic chord, $\mathrm{m}$.

$D_{\text {fan }} \quad$ Fan tip diameter, $\mathrm{m}$.

$h \quad$ Specific enthalpy, $\mathrm{J} \mathrm{kg}^{-1}$.

$M \quad$ Mach number.

$\dot{m} \quad$ Mass flow rate corrected to sea-level conditions, $\mathrm{kg} \mathrm{s}^{-1}$.

$p \quad$ Pressure, $\mathrm{Pa}$.

$r \quad$ Radius, $\mathrm{m}$.

$\tilde{r} \quad$ Span fraction $=\frac{r-r_{\text {hub }}}{r_{\text {cas }}-r_{\text {hub }}}$.

$s \quad$ Specific entropy, $\mathrm{J} \mathrm{kg}^{-1} \mathrm{~K}^{-1}$.

$T \quad$ Temperature, $\mathrm{K}$.

$U \quad$ Blade speed $=\Omega r, \mathrm{~ms}^{-1}$.

$V \quad$ Velocity, $\mathrm{ms}^{-1}$.

W Rotor-relative velocity, $\mathrm{ms}^{-1}$.

$\dot{W}_{x} \quad$ Shaft work input, $\mathrm{J} \mathrm{s}^{-1}$.

$X_{G} \quad$ Gross thrust, $\mathrm{N}$.

$y^{+} \quad$ Dimensionless wall distance.

$\alpha \quad$ Absolute flow angle $=\tan ^{-1} \frac{V_{\theta}}{V_{x}}$.

$\beta \quad$ Relative flow angle $=\tan ^{-1} \frac{V_{\theta}-U}{V_{x}}$.

$\gamma \quad$ Ratio of specific heat capacities $=1.4$.

$\Delta \quad$ Change in value of the following property.

$\eta_{\text {fan }} \quad$ Fan isentropic efficiency.

$\theta \quad$ Cylindrical polar tangential coordinate.

$\rho \quad$ Density, $\mathrm{kg} \mathrm{m}^{-3}$.

$\phi \quad$ Flow coefficient $=\frac{V_{X}}{U}$.

$\chi \quad$ Blade metal angle.

$\Omega \quad$ Rotor angular velocity, $\mathrm{s}^{-1}$.

$\omega \quad$ Vorticity, $\mathrm{s}^{-1}$.

$\tilde{\omega} \quad$ Normalised streamwise vorticity $=\frac{(\underline{\omega} \bullet \underline{W})}{W} \frac{c_{\text {tip }}}{\bar{W}_{\text {in }}}$.

Abbreviations

3D Three-dimensional.

ADP Advanced Ducted Propulsor.

BL Boundary Layer.

CFD Computationl Fluid Dynamics. 
NASA National Aeronautics and Space Administration.

PS Pressure Surface.

RANS Reynolds-averaged Nacvier Stokes.

VPF Variable Pitch Fan.

\section{Subscripts}

aa Area-averaged (spanwise) value.

$c \quad$ Value corrected to sea level condtions.

cas Casing value.

des Cruise design value.

fs $\quad$ Free-stream value.

hub Hub section value.

in Blade row inlet value.

LE Leading edge value.

ref Reference value.

rel Value in the local rotor relative frame.

TE Trailing edge value.

tip Tip section value.

TO Take-off design value.

$x \quad$ Value in the axial direction.

$\theta \quad$ Value in the direction tangential to axial and radial axes.

\section{Introduction}

Variable Pitch Fans (VPFs) address two obstacles to increasing the efficiency of civil aircraft propulsion: the requirement for extended fan stable operating range and the burden of nacelle-mounted thrust reversers.

Increasing the propulsive efficiency of a turbofan requires a reduction in Fan Pressure Ratio (FPR). However, as design FPR reduces the separation between the cruise and take off working lines increases and at low flight speeds the fan approaches its stall or flutter stability limit. Variable Pitch extends the stable operating range of a fan and allows for the separation in working lines.

Decreasing the design FPR increases installed weight and drag due to the associated rise in fan diameter. Additional weight is added for a VPF due to the variable pitch mechanism and gear system between the fan and low pressure turbine. This could be offset by eliminating the need for heavy cascade-type thrust reversers in the bypass duct. To allow this, a VPF must provide sufficient decelerating force when repitched to reverse the direction of the bypass flow.

The fuel burn benefit of a VPF turbofan therefore depends on its reverse thrust capability. This requires understanding of the fan stage aerodynamics at conditions far from design operation.

Figure 1 is a diagram showing the two ways a fan stage can be operated in reverse thrust: repitching through flat, or fine, and through feather, or stall. The flat direction requires that the blade solidity is everywhere less than one, maintains the same leading edge to the incoming flow and reverses the sense of camber. The feather direction does not restrict blade 


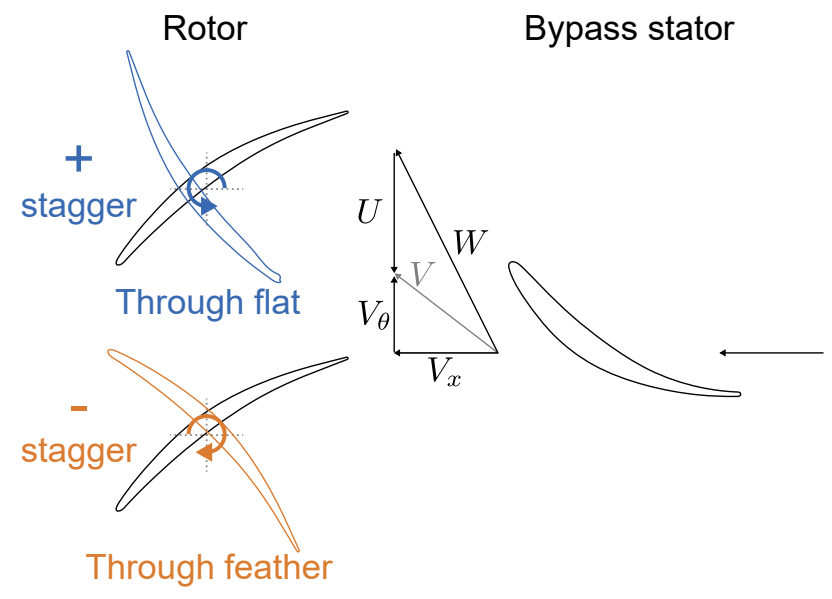

Fig. 1. Diagram of fan blade repitching for reverse thrust

solidity, employs the trailing edge as the leading edge in reverse and maintains the sense of camber. Note that in both cases the fan rotation direction is the same as for forward thrust operation. The flow enters the fan stage aft of the rotor, through the nozzle and bypass duct in a current turbofan configuration. The bypass stator thus imparts swirl against the direction of rotation, acting to increase rotor incidence.

Past experimental investigations form the basis of understanding of fan reverse thrust. NASA stages 51B and 55 [1,2] consisted of a stator row receiving uniform inlet flow and a variable pitch rotor. A region of low momentum, recirculating flow, present in all test cases, was observed to cover up to $40 \%$ of the passage from the hub downstream of the rotor. Reverse stagger settings closed the hub sections such that they completely blocked the passing of oncoming flow. Rotor 51B had a solidity everywhere less than 1 across the span so could be repitched through flat, but this resulted in lower mass flow, work and thrust than through feather. The inverse camber distribution caused suction surface separation from the leading edge, reducing the operating range due to stall. The highest work input and thrust values were universally observed at stagger settings through feather.

The NASA Quiet Clean Short-Haul Experimental Engine (QCSEE) under-the-wing VPF scale test rig was run in reverse through flat and feather settings [3,4]. For reverse thrust operation, discrete working lines existed for each pitch setting, attributed to changes in effective exit flow area with the varying extent of the hub recirculation region, and with varying levels of exit swirl. The maximum gross thrust estimated was $43 \%$ of the take-off value, at the highest loading and the most open fan stagger setting.

Further research has placed importance on installed performance over understanding of fan stage aerodynamics. The QCSEE full scale demonstrator failed to match the thrust produced by the scale fan, and fell below the target of $35 \%$ of take-off thrust [5]. Shortcomings in bypass nozzle inlet pressure recovery were cited for the lower thrust. Reemsnyder and Sagerser [6] studied another low FPR fan in reverse operation with forward wind-tunnel velocity and observed that the engine external flow field influenced the working line. A higher mass flow working line was apparent for low tunnel velocities, where the expelled jet penetrated far into the oncoming flow, with a distinct jump to a lower mass flow working line when the jet recirculated over the nacelle. The latter flow field was also observed for the NASA/Pratt \& Whitney 17 in. Advanced Ducted Propulsor, which is sketched based on Laser Doppler Velocimetry data in Fig. 2 [7].

This paper aims to improve our understanding of fan stage reverse thrust aerodynamics, by modelling a low FPR VPF using 3D CFD. The test case used is the NASA Advanced Ducted Propulsor. The computational method is first demonstrated 


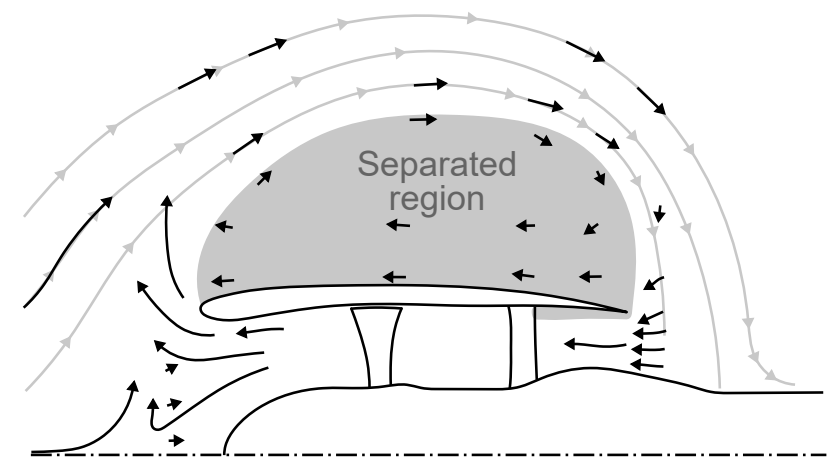

Fig. 2. Global reverse thrust flow field sketched from laser Doppler velocimetry [7] for the Advanced Ducted Propulsor 17 in. Fan

in forward thrust conditions for which experimental data is available at a range of conditions for comparison. The computational approach is then modified for reverse thrust operation. It is shown that for examining the fan stage aerodynamics in reverse thrust it is possible to use a reduced computational domain. This is then applied to obtain reverse thrust results for a range of fan speeds and pitch settings. The paper presents the flow field in reverse thrust and identifies key flow features that determine the stage performance. The results are then used to quantify the various loss sources in reverse thrust and to understand how these vary with operating point.

To the authors' knowledge, this paper is the first detailed examination of reverse thrust aerodynamics of a fan stage using CFD. It isolates the key flow features detrimental to performance for a VPF operating in this mode and quantifies their variation with operating point. In particular, it shows how a large portion of the rotor is subject to high negative incidence leading to a large pressure surface separation with associated loss and blockage. It also demonstrates how steady 3D CFD with a suitable choice of mesh, boundary conditions and domain can be used effectively to examine reverse thrust operation.

\section{Advance Ducted Propulsor Test Case}

The Advanced Ducted Propulsor (ADP) fan stage test rig was developed to investigate the aerodynamic, acoustic and structural performance of a VPF representative of future low FPR designs. The scaled fan used in this study was designated Fan 1, tested in the NASA Glenn 9-by-15 ft wind tunnel (Fig. 3). Stage design was detailed by Hobbs et al. [8], with design parameters for cruise and take-off operating points summarised in Tab. 1. The contrast between the two conditions was in the stagger setting and inlet passage Mach number. As the working line moved to a lower mass flow at take-off, the blades were closed to reduce the loading coefficient and increase stall margin. The hub-to-tip ratio was greater than that of current axial fans to accommodate the variable pitch mechanism and potential gearing.

\subsection{Instrumentation}

Forward operation measurements were taken with a flight intake and nozzle at wind-tunnel Mach numbers up to 0.20 , and at lower tunnel speed with a bellmouth intake and variable bypass nozzle to map fan working lines and constant speed characteristics. Experimental and calculation procedures are detailed by Jeracki [9], and an outline of the bellmouth configuration is given by Fite [10] for a later design of fan stage.

For all data presented, upstream total conditions were averaged from four readings in the free stream, approximately 4 fan diameters forward of the rotor stacking axis. The positions of the downstream probes are shown in Fig. 4. Four rakes upstream of the bypass stator with ten radially spaced total pressure and temperature probes upstream of the bypass stator at 
Table 1. NASA Advanced Ducted Propulsor performance summary

\begin{tabular}{|c|c|c|}
\hline & Cruise & Take-off \\
\hline Fan pressure ratio & 1.29 & 1.28 \\
\hline$M_{\text {in }}$ & 0.62 & 0.50 \\
\hline Rotor inlet tip $M_{r e l}$ & 1.0 & 0.94 \\
\hline Rotor tip Reynolds number & $2.0 \times 10^{6}$ & $1.9 \times 10^{6}$ \\
\hline Rotor stagger setting & $0^{\circ}$ & $9^{\circ}$ \\
\hline Rotor hub-to-tip ratio & \multicolumn{2}{|c|}{0.426} \\
\hline Fan diameter / m & \multicolumn{2}{|c|}{0.559} \\
\hline
\end{tabular}

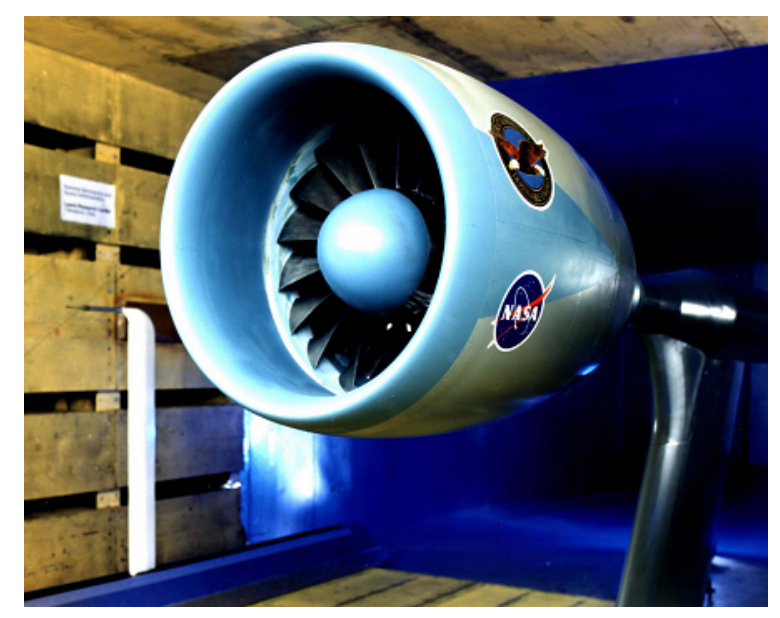

Fig. 3. NASA Advanced Ducted Propulsor test rig [9]

station F2b, and two radial rakes of five probes upstream of the core stator at F2c, were circumferentially averaged to produce rotor downstream radial distributions for the bypass and core ducts respectively. For the inlet boundary layer comparison, two radial total pressure and temperature rakes were positioned 1.4 rotor tip chords upstream of the rotor stacking axis, labelled F1. The boundary layer rakes were removed for all other data points.

Area-averaged total pressure and temperature ratios are the mean of the ten bypass values, as the probes were radially spaced for equal area.

\section{Method}

Calculations presented in this paper were carried out using TURBOSTREAM, a 3D Reynolds-averaged Navier-Stokes (RANS) finite volume method solver working on multiblock structured hexahedral meshes, designed and validated for turbomachinery problems [11]. A Spalart-Allmaras turbulence model was employed, treating all surface boundary layers as turbulent, with the inlet ratio of turbulent to laminar viscosity set to 0.2 to match reported levels [12]. Wall friction was 
calculated using wall functions for the laminar sublayer and allowing flow to slip at the wall, with $y^{+}$values on every surface below 5. Meshes were produced in NUMECA IGG/AutoGrid5 [13].

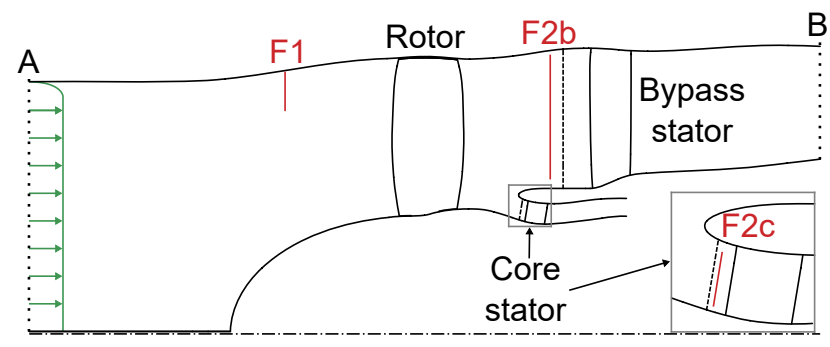

Fig. 4. ADP computational domain for forward operation. Mixing planes are drawn as dashed lines

\subsection{Comparison to Experimental Data in Forward Operation}

The simplified forward operation computational domain, displayed in Fig. 4, modelled the fan rig with bellmouth intake and variable bypass nozzle. The bypass duct extended $1.0 \times r_{\text {cas }}$ downstream of the bypass stator trailing edge, and the core duct $0.6 \times r_{\text {cas }}$ downstream of the core stator trailing edge. Planes A and B correspond to the positions of the inlet and bypass nozzle outlet for forward operation, and will be referred to in later sections. Mass flow rate in the core and bypass ducts was set by simple radial equilibrium pressure outlets.

The rotor blade row mesh consisted of 4.2 million nodes, with 165 radial divisions in the passage, 25 in the tip gap and 21 in the hub penny gaps. The bypass and core stator meshes had 2.1 million and 0.87 million nodes respectively. The casing profile was spherical above the rotor, allowing constant clearance. A clearance of $0.9 \%$ of rotor leading edge span was used at take-off rotational speed. The variation of clearance and rotor untwist with rotational speed, were modelled as described by Tweedt for the same test case [12]. The hub clearance was approximated as axisymmetric and invariant with speed, with the meridional geometry specified by Tweedt with leading edge and trailing edge clearances of $0.2 \%$ and $0.3 \%$ of span respectively.

At plane A, the inlet boundary layer total pressure profile was set empirically. For a given mass flow rate, the inlet plane Mach number, corrected for the blockage of the boundary layer, was used to calculate the free-stream static pressure at which the casing wall total pressure was set, through the variable $k_{\mathrm{p}}$, in Equation 1 . The reference pressure $p_{\text {ref }}$ was the free-stream inlet total pressure.

$$
k_{\mathrm{p}}=\left(1-\frac{p_{\mathrm{fs}}}{p_{\text {ref }}}\right)=\left(1-\left(1+\frac{\gamma-1}{2} M^{2}\right)^{-\frac{\gamma}{\gamma-1}}\right)
$$

This profile was then calculated from the inlet span fraction $\tilde{r}$ and the empirical constant $k_{\mathrm{bl}}$ through the hyperbolic function in Equation 2. The wall from the inlet to station F1 was viscous. Taking $k_{\mathrm{bl}}$ as a constant for all mass flow rates produced a good match to the total pressure readings taken at station F1, presented in Fig. 5.

$$
\frac{p_{0}(\tilde{r})}{p_{\text {ref }}}=1-2 k_{\mathrm{p}}\left(1-\frac{\tanh \left(k_{\mathrm{bl}}(\tilde{r}+1)\right)-\tanh \left(k_{\mathrm{bl}}(\tilde{r}-1)\right)}{2 \tanh k_{\mathrm{bl}}}\right)
$$

Figure 6 compares the total pressure ratio and isentropic efficiency of rig data to the CFD model. Total pressure and temperature values were extracted from the CFD domain at station F2b, and averaged consistently with the experimental 


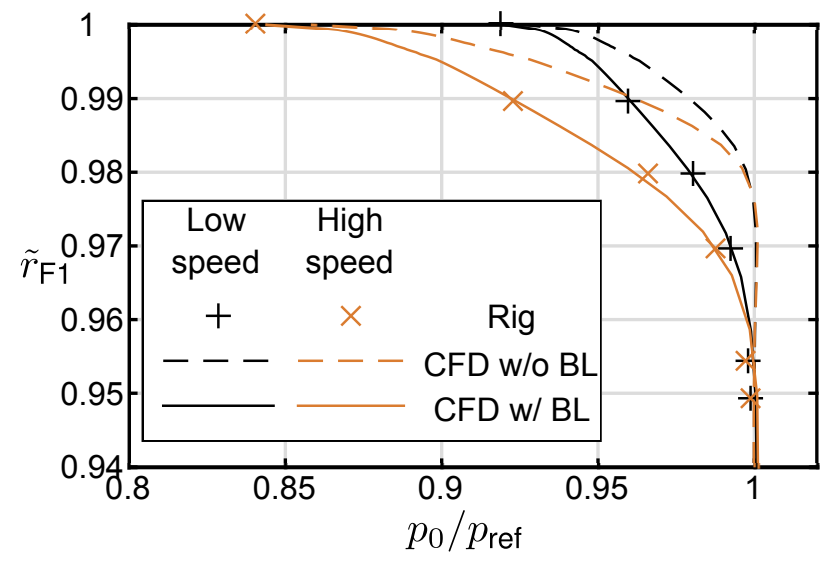

Fig. 5. Comparison of measured and computed inlet boundary layer profiles

method. The value and trend of pressure ratio about the working line was matched at all speeds. However, the CFD consistently predicts higher work input at all operating points and thus higher averaged exit total temperatures than the experiments leading to lower isentropic efficiencies.

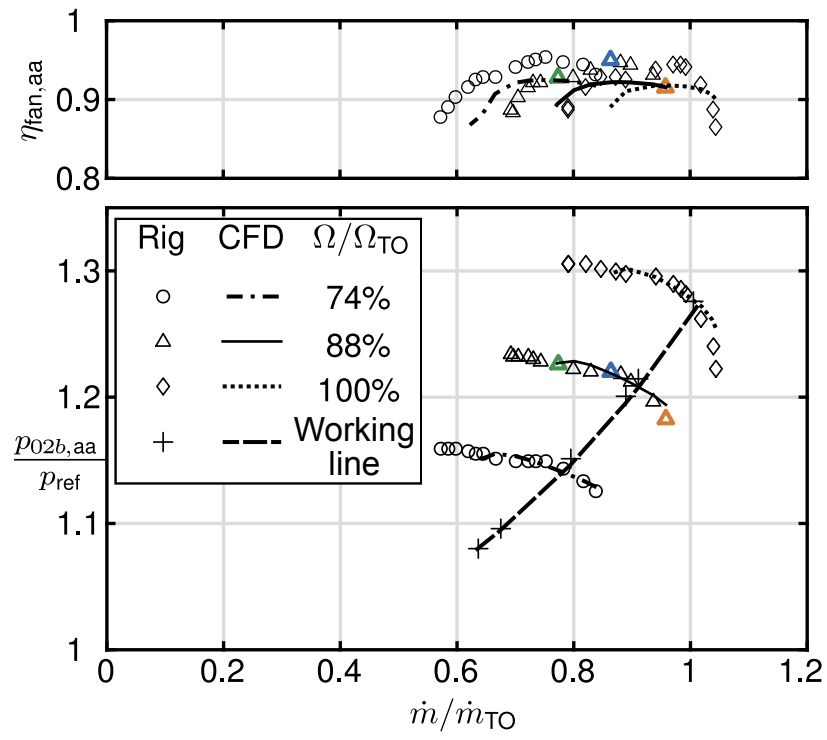

Fig. 6. Measured and computed bypass characteristics

Comparison of the radial total pressure and temperature profiles at stator inlet planes F2c and F2b is made in Fig. 7. CFD and experimental conditions were matched in corrected mass flow rate and bypass ratio, for three points at $88 \%$ take-off rotational speed: at low, mid and high flow coefficients $\phi$. Profiles were matched well at the middle and low flow coefficient, although higher temperature ratios were predicted in the CFD at the outer-most probe location. CFD profiles were higher in both pressure and temperature at high flow coefficients for $88 \%$ and $100 \%$ speed when comparing points of equal measured and computed corrected inlet mass flow rate and bypass ratio. 

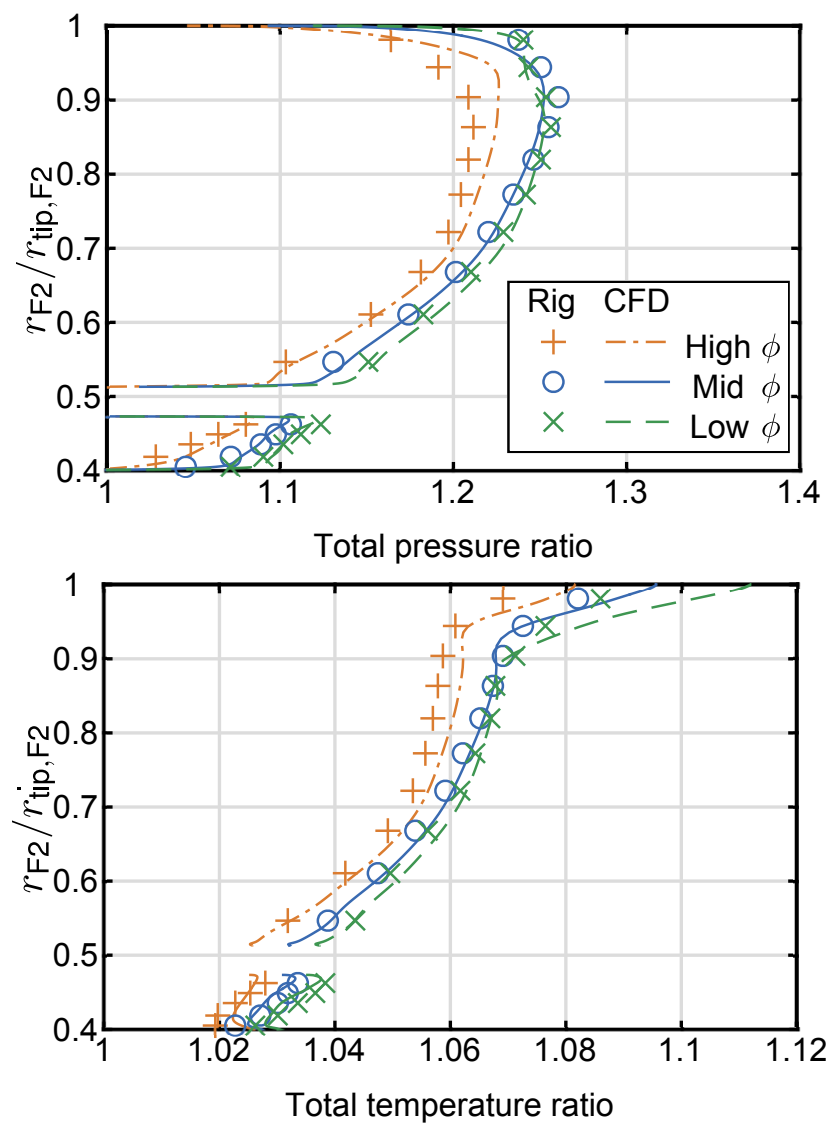

Fig. 7. Measured and computed station $\mathrm{F} 2$ radial profiles of total pressure and temperature ratios at $88 \% \Omega_{\mathrm{TO}}$

\subsection{Reverse Thrust Calculations}

For reverse thrust calculations, the axial and tangential mesh resolution was maintained from the forward operation method, but the hub penny gaps were not modelled, reducing the rotor spanwise divisions to 153 and leaving 3.7 million nodes in the rotor passage. Figure 8 shows the contrast in stagger between hub and tip sections as the blade is repitched through feather. High-stagger mesh topology allowed acceptable orthogonality of cells across the span, and free-stream mesh resolution was limited by the number of cells across the narrow throat at the hub. Solver settings were identical to those used for forward thrust calculations.

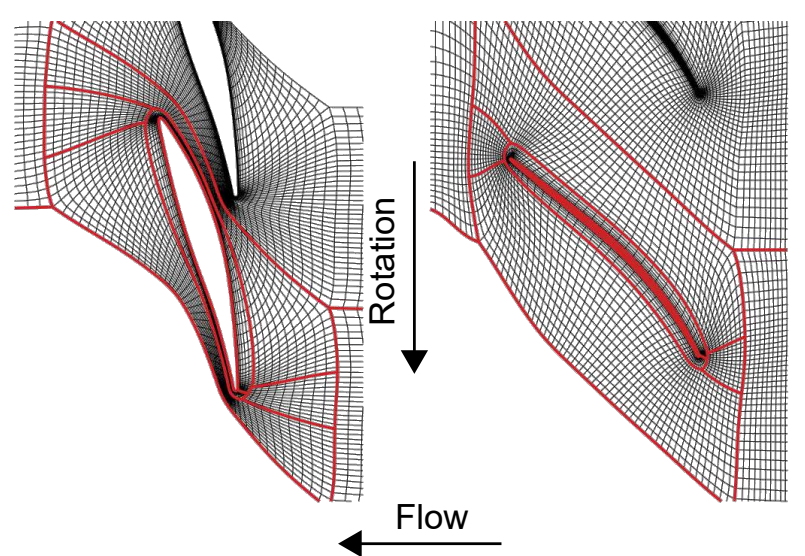

Fig. 8. Reverse thrust blade-to-blade mesh topology at the hub (left) and casing (right), repitching through feather 
The inlet flow to the bypass duct was modelled using uniform total pressure and temperature, equal to free-stream reference conditions, and zero swirl at plane A from Fig. 4. As the observed bypass duct flow in Fig. 2 indicates, there will, in reality, be pressure loss from the free-stream to the modelled inlet plane. This total pressure loss and distortion was excluded in this study to generate upper bound estimates of fan stage performance. Figure 9 illustrates the two configurations employed to model the domain downstream of station 3.

The first configuration, drawn in Fig. 9(a), modelled a free-stream cylinder, 20 fan diameters forward and aft of the rotor stacking axis, and 20 diameters radially outward from the axis of rotation. Forward flight velocity was simulated by setting uniform reference total conditions at the inlet, and an outlet pressure set at the maximum radius and observing simple radial equilibrium. The free-stream boundary allowed flow to exit at the outlet pressure or enter with inlet total properties, with the flow direction set by the calculated local flow angle. This configuration was employed to model the outlet conditions of the engine with higher fidelity and estimate fan working lines.

The second, ducted outlet configuration, pictured in Fig. 9(b), modelled the same domain axially aft of station 3, but ducted the flow with converging area axially forward. This allowed the outlet back pressure to be throttled to map the constant speed characteristics of the fan, and reduced the convergence time relative to simulations using the low Mach number freestream domain. The outlet area was reduced to ensure outflow across the exit, preventing the recirculating region from extending to the outlet plane.

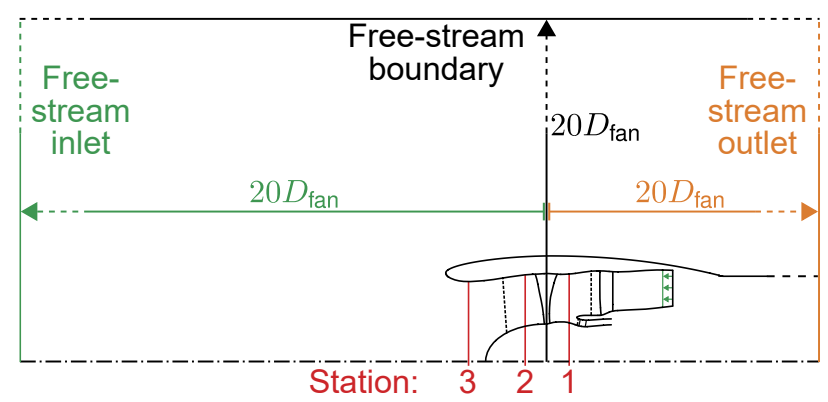

(a) Free-stream cylinder domain

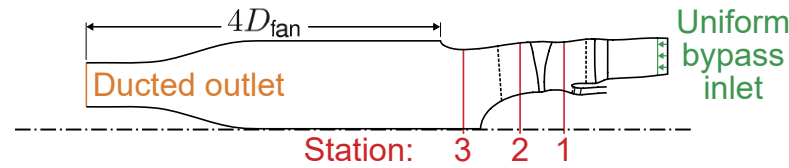

(b) Ducted outlet domain

Fig. 9. Boundary conditions for reverse thrust calculation, free-stream boundaries not to scale

For the purpose of this study, differences in the engine-external flow field were acceptable if no significant effect was observed in fan stage performance. To assess the effect for the two boundary conditions, spanwise profiles of mass flux per unit area and total and static pressure at the rotor outlet station 2 are plotted in Fig. 10. For the given operating point, the rotor mass flow rate was matched within $0.07 \%$. The profiles generated by the two boundary conditions are effectively equal, suggesting independence of fan stage performance from choice of domain configuration. 


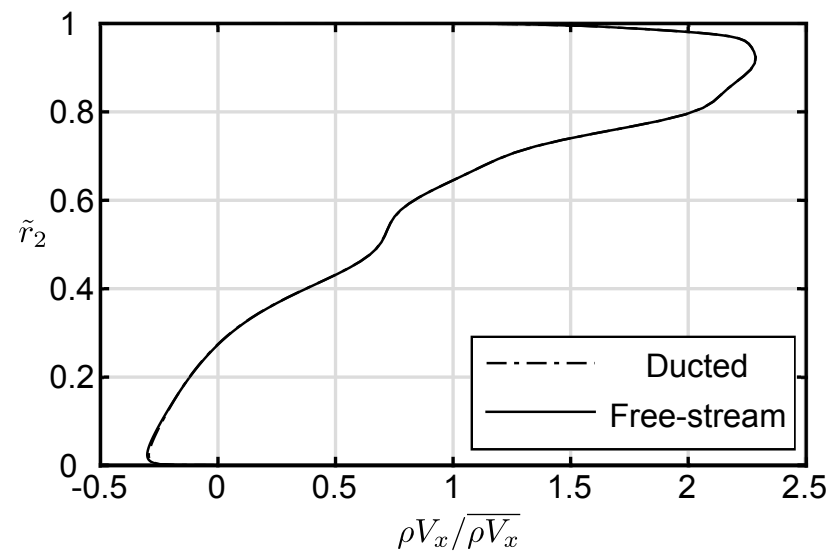

(a) Normalised mass flow per unit area

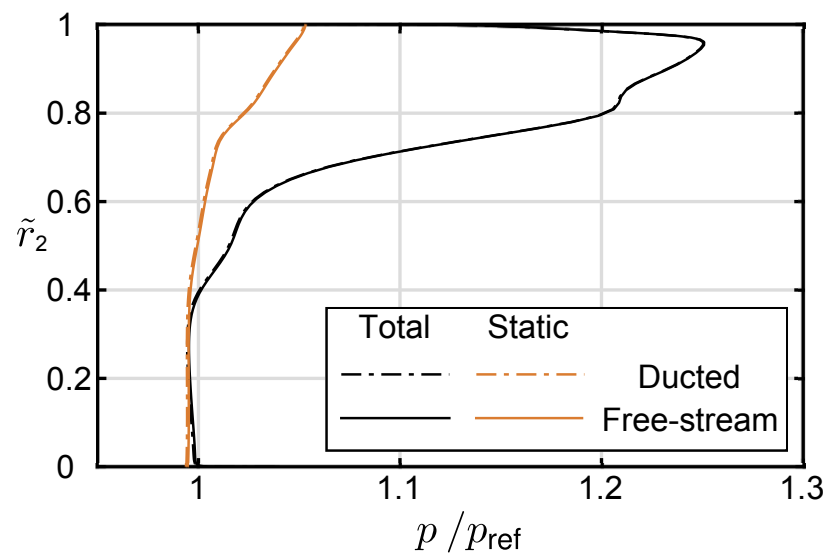

(b) Total and static pressure ratio

Fig. 10. Rotor outlet profiles for calculations with equal rotor mass flow rate for the nozzle and free-stream cylinder domains at $-92^{\circ}$ stagger and $88 \% \Omega \Omega_{\mathrm{TO}}$

\subsection{Note on the Extension of the Method}

Reverse thrust is an extreme off-design condition, for which past experimental and the current computational studies exhibit much greater regions of separated and free-shear flow than conventional (largely-attached) forward flow operation. The authors acknowledge the potential inaccuracy of prediction of turbulent viscosity for RANS solvers in free-shear flows, affecting flow loss and blockage, especially within the rotor passage. Large flow separations could be highly unsteady which may effect differences in time-averaged fan stage performance from the RANS prediction. However, the approach can be used to capture the 3D flow structure through the fan stage in reverse thrust and to predict trends in the loss sources, as demonstrated in the following sections.

\section{Fan Stage Performance}

Two rotor stagger settings through feather were selected to demonstrate the variation of key flow features and performance: at $-92^{\circ}$ and $-86^{\circ}$. The $-86^{\circ}$ case operates at high loading, and opening the blade further would push the tip sections towards stall on the working line. Loading is reduced at the more closed $-92^{\circ}$ setting, where work input is limited by increased rotor outlet blockage and the associated loss in flow capacity. 
Ducted and free-stream cylinder domain calculations were run to generate constant speed characteristics and approximate working lines respectively. Figure 11 plots the ducted domain results with open markers, and those for the free-stream domain labelled 'working line' with dashed lines and + symbols. The x-axis plots corrected mass flow rate at rotor exit, with reverse thrust cases designated negative values, and the $9^{\circ}$, take-off stagger setting forward operation cases with positive mass flow. FPR and $\eta_{\text {fan }}$ use mass-averaged total enthalpies and total pressures averaged to conserve entropy and total enthalpy, for the rotor outlet station 2 in reverse, and for stage outlet in forward operation.

As observed for test rigs in literature, each stagger setting has independent working lines. The $-92^{\circ}$ cases have a larger fraction of span taken up by a hub recirculation (see Fig. 12) reducing the effective flow area at engine exit, and higher outlet swirl, acting to increase the effective back pressure. This results in the higher total pressure rise at the same mass flow rate. This raised working line places operation at higher efficiency, and the $-86^{\circ}$ case operates further from instability due to its lower working line.
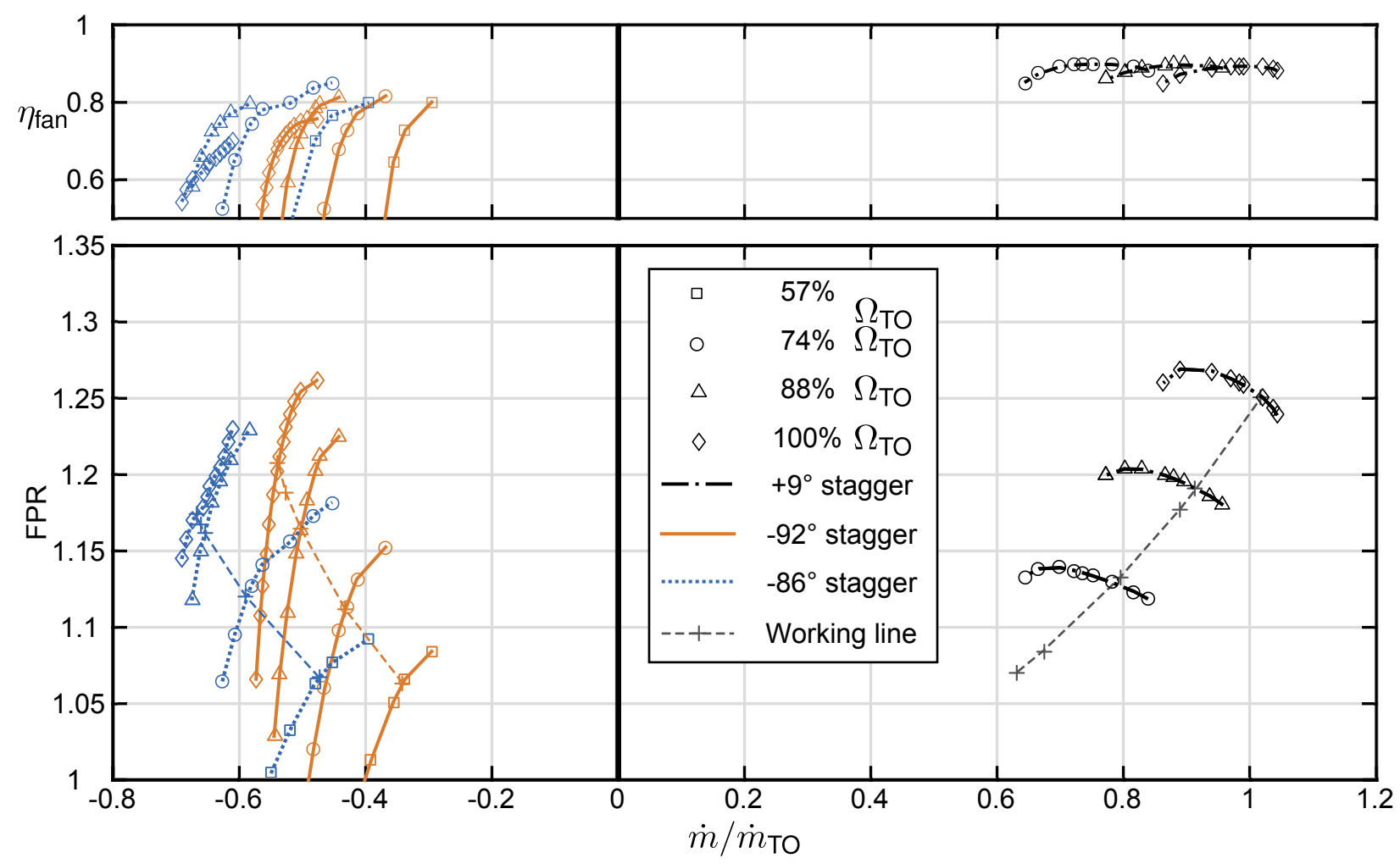

Fig. 11. Fan stage reverse total pressure ratio and isentropic efficiency characteristics

\section{The Reverse Thrust Flow Field}

Figure 12 outlines the key predicted features typical of all reverse thrust operating points. The results presented in this section are for a rotor stagger setting of $-86^{\circ}$ (through feather) from the design cruise setting and rotor speed of $88 \%$ of that at the take-off condition, on the working line.

As shown in Fig. 12(a), the undistorted inlet flow arrives axially to the bypass stator, which acts as an accelerating vane, imparting swirl against the direction of rotor rotation. The favourable pressure gradient across the blade row acts to prevent 
the suction surface boundary layer from separating, except at the trailing edge where high curvature promotes separation and increases deviation. The work lost through stator losses ranges from $4.4 \%$ to $13 \%$ of that produced through the rotor over all working line operating points.

Figure 12(b) shows the choked core stator passage in blade-to-blade view. Angular momentum imparted on the flow by the bypass stator is conserved to drive high negative incidence on the core stator. The resulting separated region off the pressure surface restricts the throat area, choking the passage and restricting mass flow. For all calculations, the core back pressure was set sufficiently low to reach the choking mass flow rate, which becomes a function only of the total mass flow rate through the bypass duct. The core mass flow rate was $57 \%$ of that at take-off conditions, for bypass duct flow rates of $74 \%$ of total take-off mass flow. Limited core flow and total pressure loss through the stator would restrict overall pressure ratio and turbine power output available to power the fan in reverse.

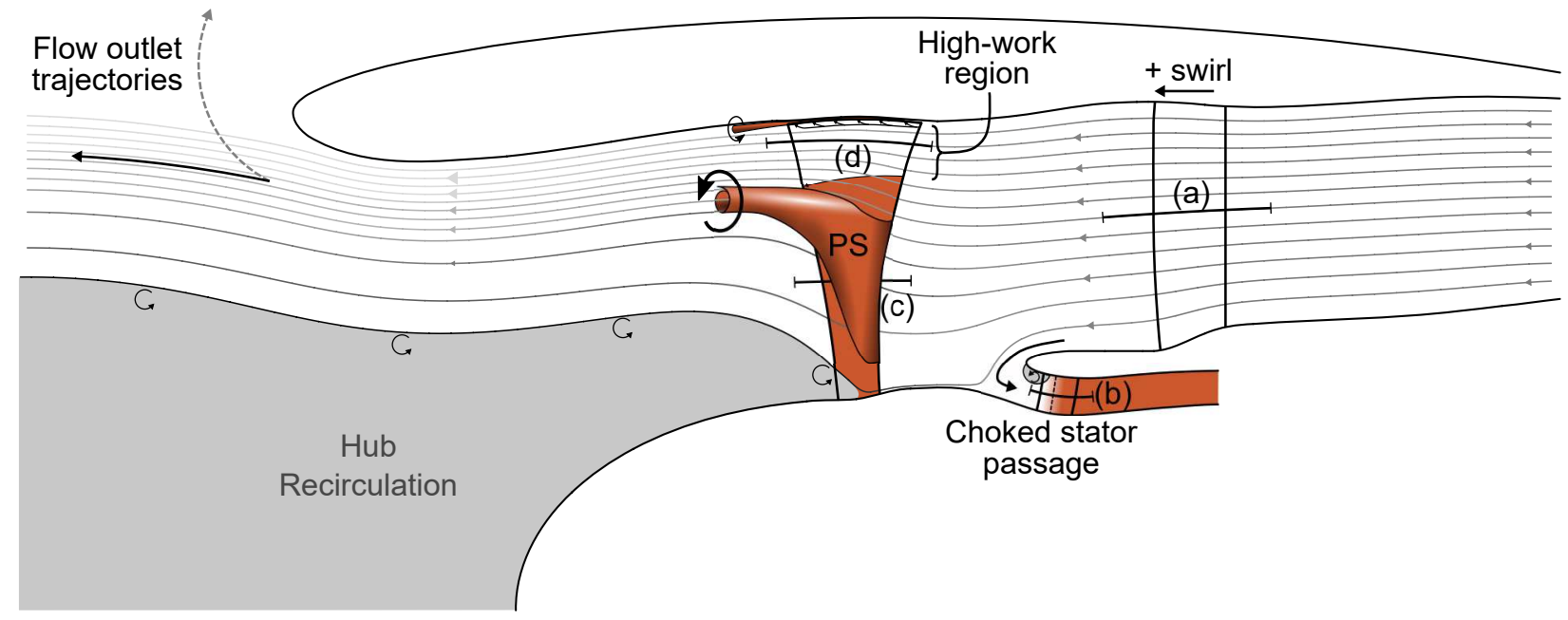

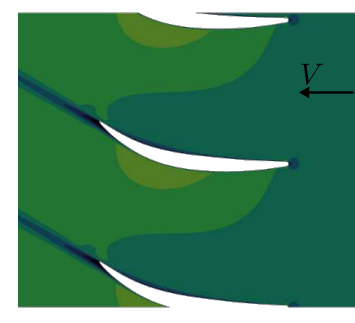

(a)

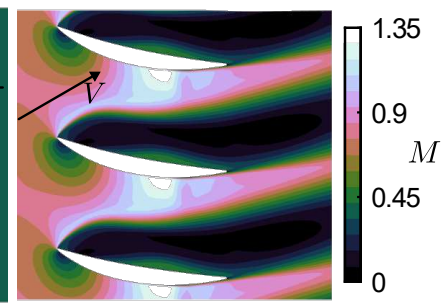

(b)

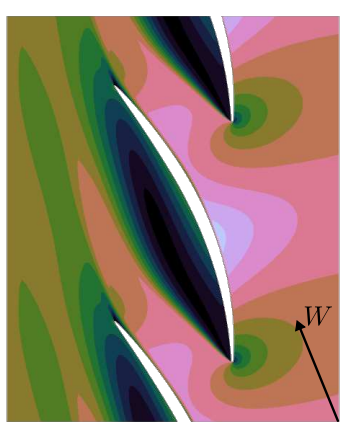

(c)

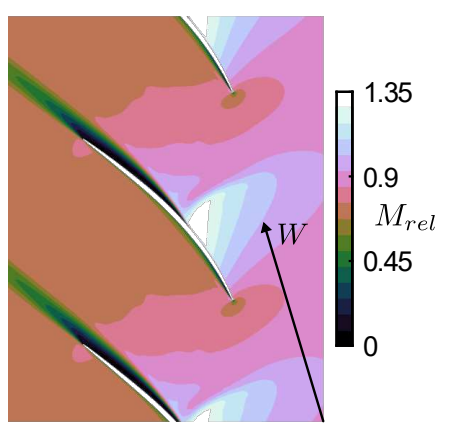

(d)

Fig. 12. Overview of the reverse thrust flow field identifying key flow features. The top diagram indicates location of the blade-to-blade cuts:

(a) bypass stator, (b) core stator. (c) rotor $40 \%$ span, and (d) rotor $90 \%$ span

Through the rotor, flow from the inner $90 \%$ of inlet span migrates radially outward, concentrating exit flow toward the casing and leaving a hub recirculation, or zero net flow, region as observed in rig tests. The hub recirculation occurs as the passage area diffuses in the meridional and tangential planes downstream of the closed-area blade passage throat. Blockage forms on the hub and the blade pressure surface where low momentum fluid experiences a strong passage-aligned pressure gradient. A negative radial pressure gradient results from this blockage as flow is diverted outward. The effective throat 
area, a function of blade stagger setting, strongly influences the passage diffusion, thus more open blade settings see reduced extent of hub recirculation. Rotor relative Mach number contours in Fig. 12(c) display the pressure surface (PS) separation associated with negative incidence and passage diffusion at $40 \%$ span. Resulting low-momentum fluid spins up to near the blade rotational speed with insufficient radial pressure gradient to overcome centrifugal effects. Thus separated pressure surface flow exits the rotor passage at a higher radius than suction surface flow entering at the same span fraction.

Sections toward the blade tip, plotted at 90\% span in Fig. 12(d), observe nominal to positive incidence and large throat clearance, allowing higher flow turning and efficiency. This feature is referred to subsequently as the high-work region. Inlet swirl means that the relative inlet Mach number is higher than for forward operation for the same mass flow rate. The chordwise-reversed camber distribution subjects the supersonic flow over the suction surface to high curvature, which is designed for subsonic diffusion. Shock normal Mach numbers are thereby enhanced, inducing boundary layer separation at lower fan speeds.

\subsection{Rotor Inlet Conditions}

Fan stage blade angles are designed for a range of forward flow conditions and their suitability for reverse thrust is not considered. When the rotor is repitched and the flow direction reversed, the radial variation of stagger angle also changes direction such that the hub section has higher stagger, or is more closed, than the tip. This can be seen in Fig. 8. The rotor reverse leading edge metal angle $\chi_{\mathrm{LE}}$ thus generally decreases with radius over the outer span, as shown in Fig. 13, which opposes the increase in blade speed. The overall effect is to move the hub towards negative incidence and the tip toward positive incidence.

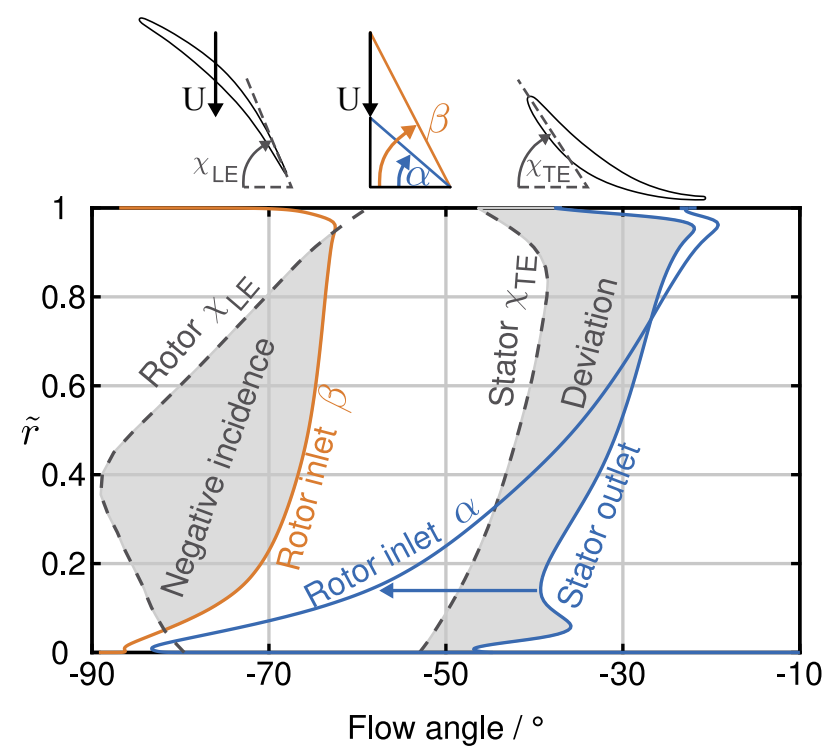

Fig. 13. Absolute and relative flow angles from bypass stator outlet to rotor inlet, at $-86^{\circ}$ stagger and $88 \% \Omega_{\mathrm{TO}}$

The development of swirl angle between the bypass stator and rotor leading edge acts to offset some of the spanwise incidence variation. Figure 13 plots the flow angle at the outlet of the stator, noting deviation angles of approximately $10^{\circ}$ discussed above. The stator reverse trailing edge angle reduces with radius, corresponding to the lower turning necessary to achieve the forward loading coefficient with increasing blade speed. Figure 12 draws the contrast in stream-tube area 
between stator outlet and rotor inlet, corresponding to a reduction in axial flow speed, to which both the increase in passage area and extraction of the core mass flow contribute. Due to this and a reduction in radius for the lower $40-50 \%$ of span, angular momentum conservation requires that the tangential velocity increases. The result is an increase in magnitude of flow angles at the rotor inlet, as seen in Fig. 13. Consequently, the rotor relative inlet angle maintains a slope to lower negative values with radius, despite increasing blade speed. Comparing the relative angle to the leading edge metal angle still reveals most of the span operates under negative incidence, but at a lower magnitude than that which would exist with a uniform absolute inlet angle.

The rotor inlet angle defines two flow regimes across the span. High negative incidence, up to $75 \%$ span for the operating condition plotted in Fig. 13, reduces potential for flow turning and induces mixing loss through pressure surface separation, seen in Fig. 12(c). Above this is the high-work region where incidence angles are closer to nominal. As the flow coefficient decreases and the incidence becomes more positive, the proportion of the span in the latter regime increases. The raised loading pushes the tip sections toward suction surface separation, such that the maximum loading is reached whilst there is still separated pressure surface lower down the span.

In summary, spanwise variation in rotor performance is tied to the increasing incidence profile with radius, established by the inlet metal angle and distribution of flow angle from the upstream stator. The bypass stator does not exhibit separated behaviour, but the core stator is subjected to choking in the current configuration due to incident swirling flow. Rotor tip sections operate with high work input, where loss increases with incidence and relative inlet Mach number. Separated pressure surface flow, due to incident flow swirling in the opposite direction, induces high loss on flow at lower inlet span fractions.

\section{Rotor Loss Characteristics}

To quantify the effect of the pressure surface separation and high-work region on rotor fan stage loss, station 2 is divided by measure of streamwise vorticity and observations of the flow field. Figure 14 plots contours of normalised streamwise vorticity $\tilde{\omega}$, defined below as the component of vorticity in the direction of relative velocity, divided by the mass-averaged inlet relative velocity $\bar{W}_{\text {in }}$ over the tip chord $c_{\text {tip }}$.

$$
\tilde{\boldsymbol{\omega}}=\frac{(\underline{\boldsymbol{\omega}} \bullet \underline{\mathbf{W}})}{W} \frac{c_{\text {tip }}}{\bar{W}_{\mathrm{in}}}
$$

Areas where the magnitude of $\tilde{\omega}$ exceed 0.5 indicate strong secondary flow, such as that due to tip clearance flow, or flow separation. There is no net work or loss over the region of recirculation at the hub, here approximated as an axisymmetric stream surface. A 'Free-stream' region (i) was assigned to cells outside the hub recirculation and below the threshold of vorticity. The 'Pressure Surface (PS) separation' (ii) was characterised by two large vortical features, one with a positive sense of rotation about the velocity vector and the other, at a higher radius, with the opposite sense. The maximum radius of the contour of negative sign was observed to approximate the boundary between flow derived from the lower radius inlet flow from the pressure surface, and the wake of the high-work outer sections. In the figure, this boundary is marked by a grey dashed line at about 77\% span. Flow area above the positive threshold was assigned 'Wake' flow of the high-work region (iii). Above $90 \%$ span, the tip clearance, or 'Casing', flow (iv) was integrated over the area below the negative threshold.

The lost work for regions (i) to (iv) was expressed as a loss of isentropic efficiency in the expression below, where the reference entropy is the mass-averaged value at rotor inlet station 1 (see Fig. 9). The lost work upstream of this plane, 


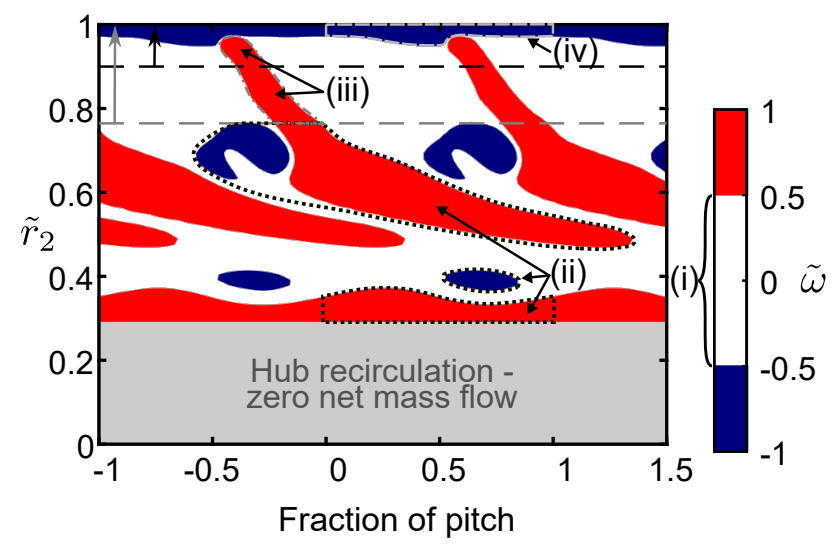

Fig. 14. Rotor outlet station 2 divided for loss integration into sections: (i)free-stream, (ii) pressure surface separation, (iii) wake, (iv) casing

labelled 'Upstream' below in Fig. 15, integrates the change in entropy between stage inlet and station 1, including the loss generated across the bypass stator and bypass mixing plane.

$$
\Delta \eta_{\mathrm{fan}, i} \approx-\frac{\bar{T}_{2}}{\dot{m}\left(\overline{h_{02}}-\overline{h_{01}}\right)} \int_{S_{i}}\left(s_{2}-\bar{s}_{1}\right) \rho V_{x} d A_{x}
$$

Figure 15 plots the fan efficiency and integrated components of lost work for working line conditions at $-92^{\circ}$ and $-86^{\circ}$ stagger. At $-86^{\circ}$ both flow coefficient and inlet Mach number are higher for the same rotor speed. Each working line operates at a different, but approximately constant flow coefficient across speeds. Below $80 \%$ speed the pressure surface separation lost work accounts for $16 \%$ of the total work at $-92^{\circ}$ and $11 \%$ at $-86^{\circ}$. This reflects the larger proportion of mass flow through this region with the more closed blade.

The largest variation with rotor speed is seen for the $-86^{\circ}$ setting in the combined casing, wake and free-stream flow which comprise the high-work region. The contribution to overall lost work grows from $13 \%$ to $22 \%$ of total work input betweeen $70 \%$ and $100 \%$ speed. This rise is a Mach number effect, when shock strength increases to influence the suction surface boundary layer to separate.

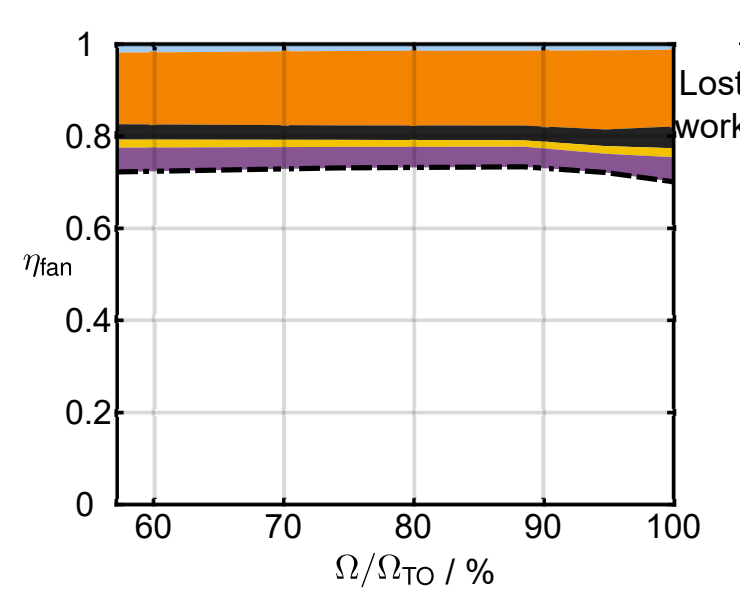

(a)

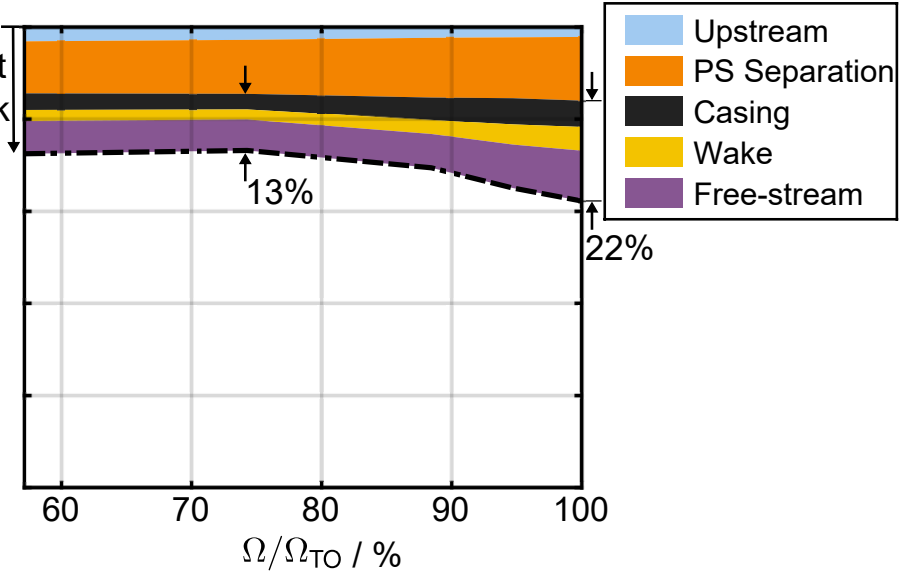

(b)

Fig. 15. Loss breakdown on the reverse working line at (a) $-92^{\circ}$ and (b) $-86^{\circ}$ stagger 


\section{Quantifying the Computed Reverse Thrust}

A consistent measure of axial thrust is defined below, representing the force applied to a stream tube originating at a static free-stream state, where the static pressure is equal to the inlet reference total pressure $p_{0, \text { ref }}$, and closed at the outlet plane: station 3 for reverse operation, or plane B (Fig. 4) for forward operation. $X_{G}$ is recognisable as the gross thrust, and is quantified here for both forward and reverse thrust cases. In the comparison of values using uniform, free-stream reference total pressure and temperature at the bypass duct inlet, it is assumed the inlet stream tube is isentropic. This produces upper bounds of thrust that would reduce with pressure loss in recovering the flow to the bypass duct, and momentum transfer from the expelled jet to inlet stream tube.

$$
X_{G}=\int_{\text {outlet }}\left[\left(p-p_{0, \text { ref }}\right)+\rho V_{x}^{2}\right] d A_{x}
$$

The magnitude of $X_{G}$, normalised by the $100 \%$ rotational speed, $9^{\circ}$ stagger take-off value, is compared at the two stagger settings across their working lines in Fig. 16. Maximum gross thrust is estimated to be $44.8 \%$ for $-92^{\circ}$ and $45.7 \%$ for $-86^{\circ}$, both at the maximum rotor speed. The higher flow $-86^{\circ}$ setting produces greater $X_{G}$ at all speeds, but the difference between the settings decreases from $90 \%$ and $100 \%$ rotor speed, corresponding to the increased loss observed in Fig. 15(b).

The comparison of shaft power normalised by that at the take-off condition highlights the translation of lost work to reduction in thrust for the $-86^{\circ}$ setting. Addition of power input beyond $90 \%$ take-off rotor speed does not enhance thrust, as high-work region losses increase.

A third working line, included in Fig. 16, was calculated at an intermediate stagger setting of $-89^{\circ}$ which has higher work input than the $-92^{\circ}$ case and does not suffer as severe a rise in high-work region lost work with rotor speed as $-86^{\circ}$ case. The result is that the setting with maximum thrust switches from $-86^{\circ}$ to $-89^{\circ}$ above $80 \% \Omega_{\mathrm{TO}}$, with a peak gross thrust value $49.0 \%$ of that at take-off at $100 \% \Omega_{\mathrm{TO}}$. The choice of stagger setting to produce the greatest thrust must achieve increased work input through opening the rotor blades, but not to the extent that the rise in high-work region loss cancels this increase.

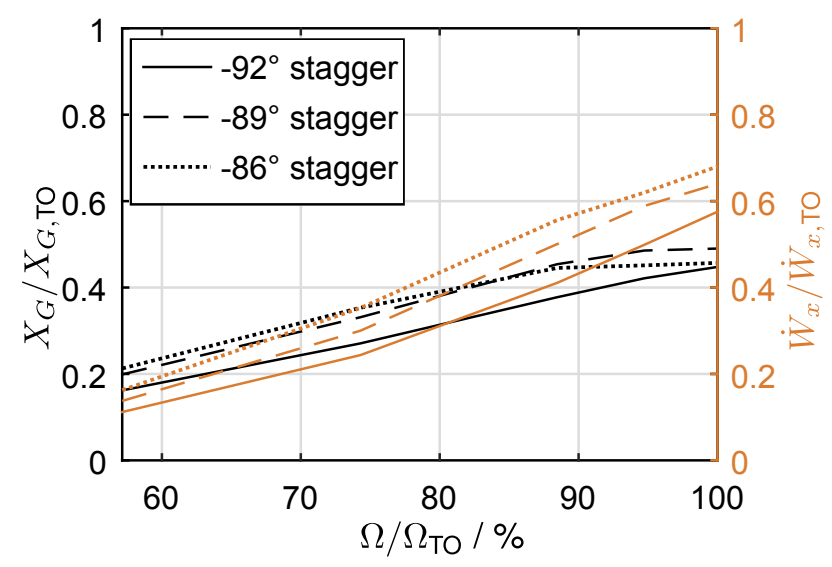

Fig. 16. Estimate of gross thrust, and work input normalised by $100 \%$ speed take-off values 


\section{Conclusions}

The flow field for a fan stage in reverse operation has been predicted using 3D CFD. The method was validated against spanwise experimental probe measurements for the ADP test case at the forward take-off rotor stagger setting and extended to simulate the fan in reverse. CFD predictions of stage performance have been found to be unchanged for a reduced domain, allowing constant speed characteristics to be mapped.

Stage performance is characterised by a high-work region towards the rotor tip and largely separated pressure surface flow over at least the inner half of rotor span. The variation in rotor leading edge angle with radius leads the tip towards positive incidence and the hub towards negative incidence, but the development of flow angle from the swirl added by the bypass stator is shown to mitigate this effect, particularly at the hub. High hub blockage leads to a recirculating region downstream of the rotor. Reductions in flow coefficient at fixed stagger setting improve the performance of the lower radius flow under negative incidence, and increase loading at the tip, pushing the high-work section towards stall.

The pressure surface separation flow is found to contribute the most to stage loss, or inefficiency, for the closed blade $-92^{\circ}$ stagger setting, accounting for $16 \%$ of total work input. This loss is less significant at a more open blade setting, with higher throat area and flow coefficient, for which the high-work region losses grow with relative inlet Mach number as high leading edge curvature generates shock normal Mach numbers high enough to separate the suction surface boundary layer.

Working line gross thrust reaches $49 \%$ of the take-off value at $100 \%$ take-off rotor speed at $-89^{\circ}$ stagger. This value represents an upper bound of thrust potential, which will reduce with pressure loss and distortion in the bypass duct. Increased reverse thrust is achieved with raised loading and power input at more open stagger settings, but avoiding increased loss in the high-work region at high speed.

\section{Acknowledgements}

The authors would like to thank Rolls-Royce plc for funding and permission to publish, EPSRC for funding through the Centre for Doctoral Training in Gas Turbine Aerodynamics, and Turbostream for the use of their solver. Thanks go to Mark Wilson and Stéphane Baralon at Rolls-Royce for their support and advice, and to Professor Nick Cumpsty for his comments on the work. The ADP test case geometry and data were provided by NASA Glenn Research Centre, and thanks there go to the Acoustics Branch, as well as Brad Belcher at Rolls-Royce Corporation, for their work in facilitating the sharing of data and discussion on the subject.

\section{References}

[1] Kovich, G., and Moore, R. D., 1976. Performance of 1.15-Pressure-Ratio Fan Stage at Several Rotor Blade Setting Angles With Reverse Flow. Tech. Rep. NASA TM X-3451.

[2] Moore, R. D., Lewis, G. W., and Tysl, E. R., 1976. Performance of a Low-Pressure Fan Stage with Reverse Flow. Tech. Rep. NASA TM X-3349.

[3] Giffin, R. G., McFalls, R. A., and Beacher, B. F., 1977. Quiet Clean Short-Haul Experimental Engine (QCSEE). Aerodynamic and Aeromechanical Performance of a $50.8 \mathrm{~cm}$ (20 in.) Diameter 1.34 PR Variable Pitch Fan with Core Flow. Tech. Rep. NASA CR-135017.

[4] Advanced Engineering \& Technology Programs Department Group Engineering Division, 1977. Quiet Clean Short- 
Haul Experimental Engine. Under-the-Wind Simulation Report. Tech. Rep. NASA CR-134914, General Electric Company.

[5] Willis, W. S., 1979. Quiet Clean Short-haul Experimental Engine (QCSEE). Double-annular clean combustor technology development report. Tech. Rep. NASA CR-159473.

[6] Reemsnyder, D. C., and Sagerser, D. A., 1979. Effect of forward velocity and crosswind on the reverse-thrust performance of a variable-pitch fan engine. Tech. Rep. NASA TM-79059.

[7] Holcombe, V., 2003. Aero-Propulsion Technology (APT) Task V Low Noise ADP Engine Definition Study. Tech. Rep. NASA CR-2003-212521.

[8] Hobbs, D. E., Neubert, R. J., Malmborg, E. W., Philbrick, D. H., and Spear, D. A., 1995. Low Noise Research Fan Stage Design. Tech. Rep. NASA TM-195382.

[9] Jeracki, R. J., 2006. Comprehensive Report of Fan Performance From Duct Rake Instrumentation on 1.294 Pressure Ratio, 806 ft/sec Tip Speed Turbofan Simulator Models. Tech. Rep. NASA TM-2006-213863.

[10] Fite, E. B., 2006. Fan Performance From Duct Rake Instrumentation on a 1.294 Pressure Ratio, 725 ft/sec Tip Speed Turbofan Simulator Using Vaned Passage Casing Treatment. Tech. Rep. NASA TM-2006-214241.

[11] Brandvik, T., and Pullan, G., 2011. "An Accelerated 3D Navier-Stokes Solver for Flows in Turbomachines". Journal of Turbomachinery, 133(2), p. 021025.

[12] Tweedt, D. L., 2014. Computational Aerodynamic Simulations of an $840 \mathrm{ft} / \mathrm{sec}$ Tip Speed Advanced Ducted Propulsor Fan System Model for Acoustic Methods Assessment and Development October 2014. Tech. Rep. NASA CR-2014218129.

[13] NUMECA International, 2014. User Manual - Autogrid5 ${ }^{T M} v 9$. Brussels, Belgium. 